\title{
Conformal predictors
}

\author{
Alexander Gammerman
}

Published online: 29 August 2012

(C) Springer-Verlag 2012

\begin{abstract}
The paper introduces conformal predictors and summarises several important applications for this special issue of the journal. It includes medical diagnostics of ovarian and breast cancers; network traffic classification; classification of images obtained in a nuclear fusion device; air pollution estimation and an application for a testing of the normality assumption.
\end{abstract}

Keywords Conformal predictors - Martingales - Medical diagnostic - Air pollution estimation - Network traffic . Image classification

This special issue is devoted to the newly developed theory and applications of conformal predictors [1,2]. These machine learning methods are becoming popular since they allow to control number of errors by changing a preset significance level. They are also automatically valid in online mode. The main idea of conformal predictors is to test how well new examples fit to previously accumulated data- the training set. As usual, our data consist of a set of pairs $\left(x_{i}, y_{i}\right)$, where $x_{i}$ is an object and $y_{i}$ is a label. We assume that these data are i.i.d. data - the pairs are independent and generated by the same (but unknown) stochastic mechanism. If our task is classification - to label new examples from the test setthen we can try each label in turn, and see if the resulting sequence of $x$ 's and $y$ 's conforms the assumption. The label that in some sense fits the best to the underlying assumption would be our classification of the new example. To make this conclusion, we need to introduce a non-conformity measure (NCM), or in other words, a measure of strangeness, of the new example in comparison with the training set. This is

A. Gammerman $(\bowtie)$

Computer Learning Research Centre Royal Holloway,

University of London, Egham, Surrey TW20 0EX, UK

e-mail: alex@cs.rhul.ac.uk done by measuring the "strangeness" using $p$ values that are approximations of the well-known Martin-Löf test for randomness. In other words, we measure how "random" the new example is in respect to the accumulated data. This measure of strangeness can be easily extracted from the vast majority (if not all) known algorithms of classification or regression, such as nearest neighbours, neural networks, support vector machines (SVM) and others. This approach allows us not only just to make a prediction, but also to estimate the confidence of that prediction. Basically, if we take away from a unit the second largest $p$ value, we can estimate the confidence of the prediction: the larger the difference, the more confident we are in our prediction. Moreover, the predictions are valid in the sense that if we set up a certain significance level-say, $5 \%$ (or $95 \%$ confidence level)—we can guarantee in the online mode that we shall get no more than $5 \%$ of errors. This is a very useful feature of conformal predictors and, as we said, it allows to control the number of errors according to a preset significance level.

The current issue presents further development and various applications of the conformal predictors. The applications include network traffic, estimation of air pollution, medical diagnostics and image analysis.

In the paper by Fedorova et al., the main goal is to test the assumption of normality. We assume that the data are generated by a Gauss linear model and we want to test this assumption. In [1], a tool was developed to test the i.i.d. assumption. A similar idea has been applied here to test the assumption of normality. This is important since to guarantee validity not only as a theoretical conclusion but also for every particular dataset we have to test any accepted assumption. The testing of the normality is done by calculating some special martingales.

In the paper of Gonzalez et al., the authors present an approach that uses non-conformity measures for selecting the 
most appropriate regions for image classification problems. The one-against-rest version of SVM is used and for each class the best image region is selected after trying out a set of possible regions. The quality of each region is calculated based on the non-conformity scores of the inductive conformal prediction calibration examples. One of the results of the paper suggests that the bigger the selected area, the higher the quality of the predictions.

Dashevsky and Luo's paper considers an accurate classification of network traffic. The network resource management systems require high quality, reliable predictions with confidence measures. These reliability measures allow to optimise network performance such as delay and information loss. The paper presents Conformal Predictor and Venn Probability Machine for application in network traffic classification. Experiments on publicly available real network traffic datasets in on-line setting show that these two methods can perform well and produce reliable classifications.

Ivina et al.'s paper aims to evaluate the levels of air pollution for the Barcelona Metropolitan Region using a Ridge Regression Confidence Machine predictor. The approach gives valid interval estimates, i.e. the prediction intervals that hold the actual value of the dependent factor (label) with a given level of confidence. The results of the ridge regression estimates are compared to the results of a classic kriging.

Devetyarov et al.'s paper describes an application of Mondrian predictors-a generalization of conformal predictorsto risk assessment of ovarian and breast cancers. The analysis is based on mass spectrometry profiling of human serum samples that were collected in the United Kingdom Collaborative Trial of Ovarian Cancer Screening. The paper describes the technique and presents the results of classification (diagnosis) and the corresponding measures of confidence of the diagnostics. The main advantage of this approach is a proven validity of diagnosis and a possibility to obtain an early diagnosis of ovarian and breast cancers.

Presented in this issue papers demonstrate in various applications several very useful features of conformal predictors. In particular, conformal predictors:

- give provably valid measures of confidence;

- do not make any additional assumptions about the data beyond the i.i.d. assumption;

- allow us to estimate confidence of the prediction for individual examples;

- can be used as a region predictor, with a number of possible predicted values;

- can be used in on-line and off-line learning as well as in "intermediate" types of learning, e.g., "slow", "lazy".

Thus, the papers confirm experimentally the property of validity of conformal predictors and demonstrate that the accuracy is sufficient for many practical problems.

Acknowledgments We are greatly indebted to Professor Fionn Murtagh, of the editorial board of this journal, for his constant help and support throughout this project. We would also like to express our thanks to all referees of the papers.

\section{References}

1. Vovk, V., Gammerman, A., Shafer, G.: Algorithmic Learning in a Random World, pp. 191-197. Springer, New York (2005)

2. Gammerman, A., Vovk, V.: Hedging predictions in machine learning. Comput. J. 50(2), 151-163 (2007) 